\title{
Multicentre comparison of quantitative PCR-based assays to detect SARS-CoV-2, Germany, March 2020
}

Maximilian Muenchhoff ${ }^{1,2}$, Helga Mairhofer ${ }^{1,2}$, Hans Nitschko ${ }^{1,2}$, Natascha Grzimek-Koschewa ${ }^{1,2}$, Dieter Hoffmann ${ }^{2,3}$, Annemarie

Berger $^{2,4}$, Holger Rabenau ${ }^{2,4}$, Marek Widera ${ }^{2,4}$, Nikolaus Ackermann ${ }^{5}$, Regina Konrad ${ }^{5}$, Sabine Zange ${ }^{2,6}$, Alexander Graf ${ }^{7}$,

Stefan Krebs ${ }^{7}$, Helmut Blum ${ }^{7}$, Andreas Sing ${ }^{5}$, Bernhard Liebl5, Roman Wölfel ${ }^{2,6}$, Sandra Ciesek ${ }^{2,4}$, Christian Drosten ${ }^{2,8}$, Ulrike

Protzer $^{2,3}$, Stephan Boehm ${ }^{1,2}$, Oliver T Keppler ${ }^{1,2}$

1. Max von Pettenkofer Institute and Gene Center, Virology, National Reference Center for Retroviruses, Ludwig Maximilian University, Munich, Germany

2. German Center for Infection Research, Partner Site Munich and Associated Partner Site Charité, Berlin and Associated Partner Site Frankfurt, Germany

3. Institute of Virology, School of Medicine, Technical University Munich/Helmholtz Zentrum München, Munich, Germany

4. Institute of Medical Virology, University Hospital, Goethe University Frankfurt am Main, Frankfurt, Germany

5. Bavarian Health and Food Safety Authority, Oberschleißheim, Germany

6. Bundeswehr Institute of Microbiology, Munich, Germany

7. Laboratory for Functional Genome Analysis (LAFUGA), Gene Center, Ludwig Maximilian University, Munich, Germany

8. Institute of Virology, Charité University Medicine, Berlin, Germany

Correspondence: Oliver T Keppler (keppler@mvp.Imu.de)

Citation style for this article:

Muenchhoff Maximilian, Mairhofer Helga, Nitschko Hans, Grzimek-Koschewa Natascha, Hoffmann Dieter, Berger Annemarie, Rabenau Holger, Widera Marek,

Ackermann Nikolaus, Konrad Regina, Zange Sabine, Graf Alexander, Krebs Stefan, Blum Helmut, Sing Andreas, Liebl Bernhard, Wölfel Roman, Ciesek Sandra

, Drosten Christian, Protzer Ulrike, Boehm Stephan, Keppler Oliver T. Multicentre comparison of quantitative PCR-based assays to detect SARS-CoV-2, Germany,

March 2020. Euro Surveill. 2020;25(24): pii=2001057. https://doi.org/10.2807/1560-7917.ES.2020.25.24.2001057

Article submitted on 28 May 2020 / accepted on 17 Jun 2020 / published on 18 Jun 2020

Containment strategies and clinical management of coronavirus disease (COVID-19) patients during the current pandemic depend on reliable diagnostic PCR assays for the severe acute respiratory syndrome coronavirus 2 (SARS-CoV-2). Here, we compare 11 different RT-PCR test systems used in seven diagnostic laboratories in Germany in March 2020. While most assays performed well, we identified detection problems in a commonly used assay that may have resulted in false-negative test results during the first weeks of the pandemic.

Strategies to limit the severe pandemic and to manage coronavirus disease (COVID-19) patients strongly depend on readily available, accurate and reliable RT-PCR assays to detect the genome of the causative agent acute respiratory syndrome coronavirus 2 (SARSCoV-2) in biosamples. The first full-length SARS-CoV-2 genome sequence was made publicly available in early January 2020 [1] and, soon after, various RT-PCR assays were reported by academic laboratories, public health agencies and diagnostics companies [2-6]. Their overall performance and relative sensitivity are largely unclear.

The aim of this study was to compare the inter-laboratory and inter-method sensitivity of different RT-PCR assays by providing a blinded, frozen dilution series of a nucleic acid extract of a highly positive biosample to seven different diagnostic laboratories in Germany in March 2020.

\section{Sample preparation and study design}

Nucleic acids were pooled from multiple extractions of one SARS-CoV-2-positive stool sample using the QIAsymphony DSP Virus/Pathogen Kit (Qiagen, Hilden, Germany). This stool sample was from a 5-year-old child with COVID-19 [7] and was chosen because of high initial PCR signals and sufficient sample availability to generate large quantities of eluate for further distribution. Of note, no PCR inhibition was observed for detection of the spiked-in extraction RNA control (QuantiNova IC Probe Assays Red 650, Qiagen). A 1:10 dilution series was prepared and aliquots were labelled in a blinded fashion to be shipped on dry ice to participating laboratories in March 2020. Participants were instructed to perform the diagnostic assays used at their centre for SARS-CoV-2 detection in quadruplicate using $5 \mu \mathrm{L}$ of the aliquot per reaction. All results were reported back to the initiating laboratory (Laboratory 1) before the results were unblinded. The details of all these PCR-based assays are summarised in Table 1.

In parallel, samples were quantified using the OneStep RT-digital droplet (dd)PCR Advanced Kit for Probes (BioRad, Feldkirchen, Germany) on the BioRad QX200 platform. Primer and probe sequences were used for detection of the SARS-CoV-2 nucleocapsid gene $(N)$ as published by the Centers for Disease Control and Prevention (CDC) [2] and the envelope gene $(E)$, the RNA-dependent RNA polymerase $(R d R p)$ gene and the $N$ gene as published by Corman et al. (referred to as Charité protocol) [3] (Figure 1). 
Specifications of different molecular assays used for detection of SARS-CoV-2, Germany, March 2020 (n =11 test systems with 34 different reaction-lab combinations)

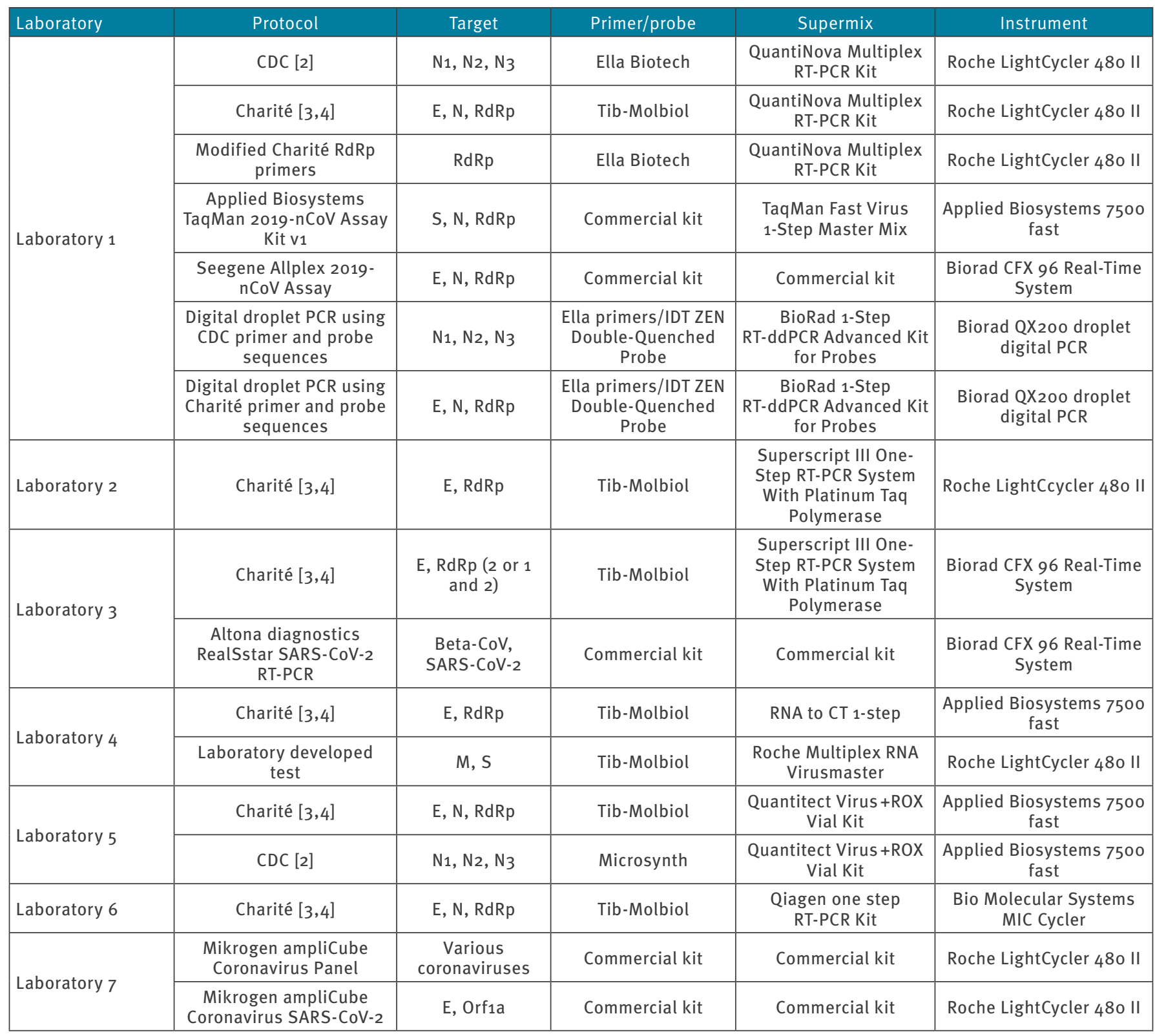

Beta-CoV: Betacoronavirus; CDC: Centers for Disease Control and Prevention; E: envelope gene; N: nucleocapsid gene; Orf: open reading frame; RdRp: RNA-dependent RNA-polymerase gene; SARS-CoV-2: severe acute respiratory syndrome coronavirus 2; WHO: World Health Organization.

Performing laboratory, assay protocol, target, manufacturer of primer/probe, PCR chemistry and instrument are indicated. 
Digital droplet PCR quantification of the distributed dilution series of nucleic acid eluate of SARS-CoV-2-positive clinical material, Germany, March 2020

A. Undiluted sample

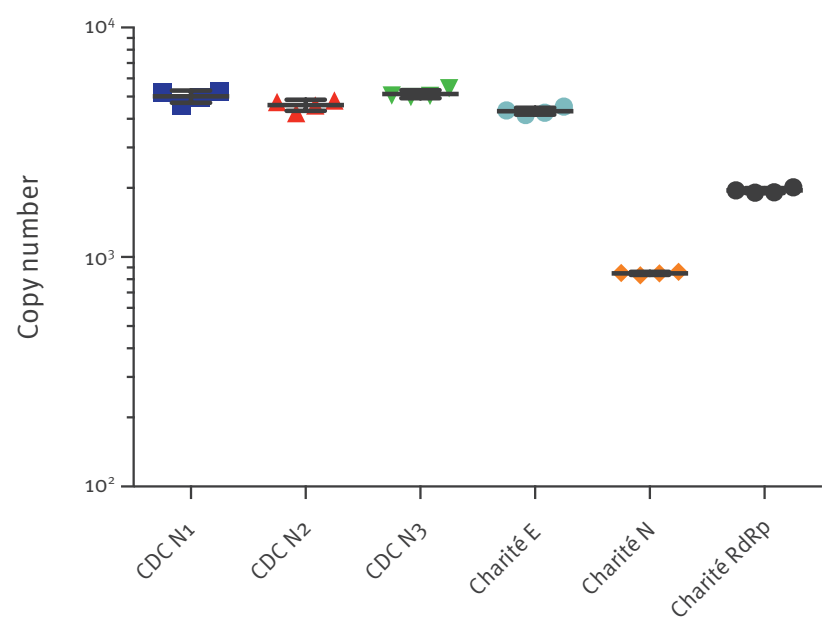

B. 1:10 dilution series

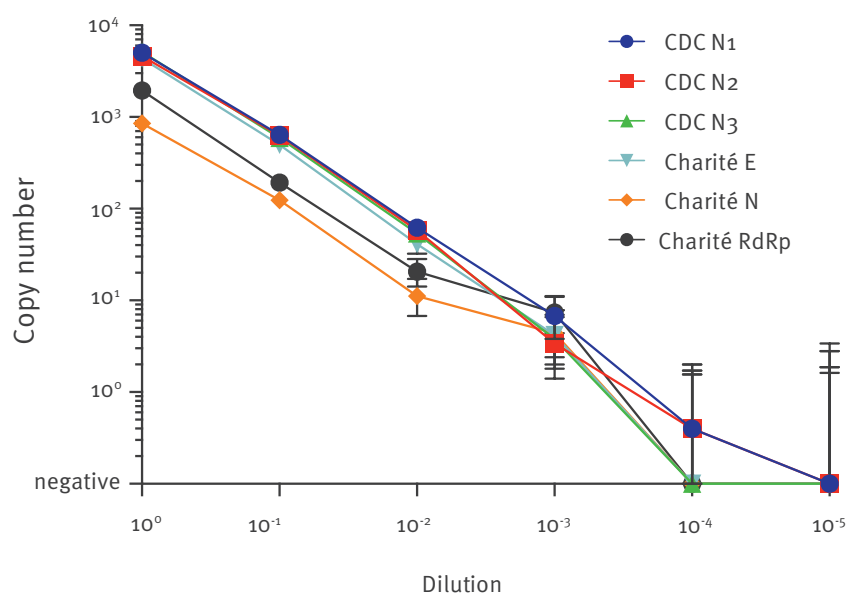

CDC: Centers for Disease Control and Prevention; E: envelope gene; N: nucleocapsid gene; RdRp: RNA-dependent RNA-polymerase gene; SARS-CoV-2: severe acute respiratory syndrome coronavirus 2.

The RNA copy numbers are indicated for each PCR reaction using either the CDC and the Charite primer/probe combinations and were measured from $5 \mu \mathrm{L}$ nucleic acid eluate. Mean values of quadruplicates are indicated by the horizontal lines (A) or symbols (B). Error bars represent the $95 \%$ Poisson confidence interval (B).

\section{FIGURE 2}

Dilution series comparing various RT-PCR assays for the detection of SARS-CoV-2 at different laboratories, Germany, March 2020 ( $\mathrm{n}=11$ test systems with 34 reaction-lab combinations)

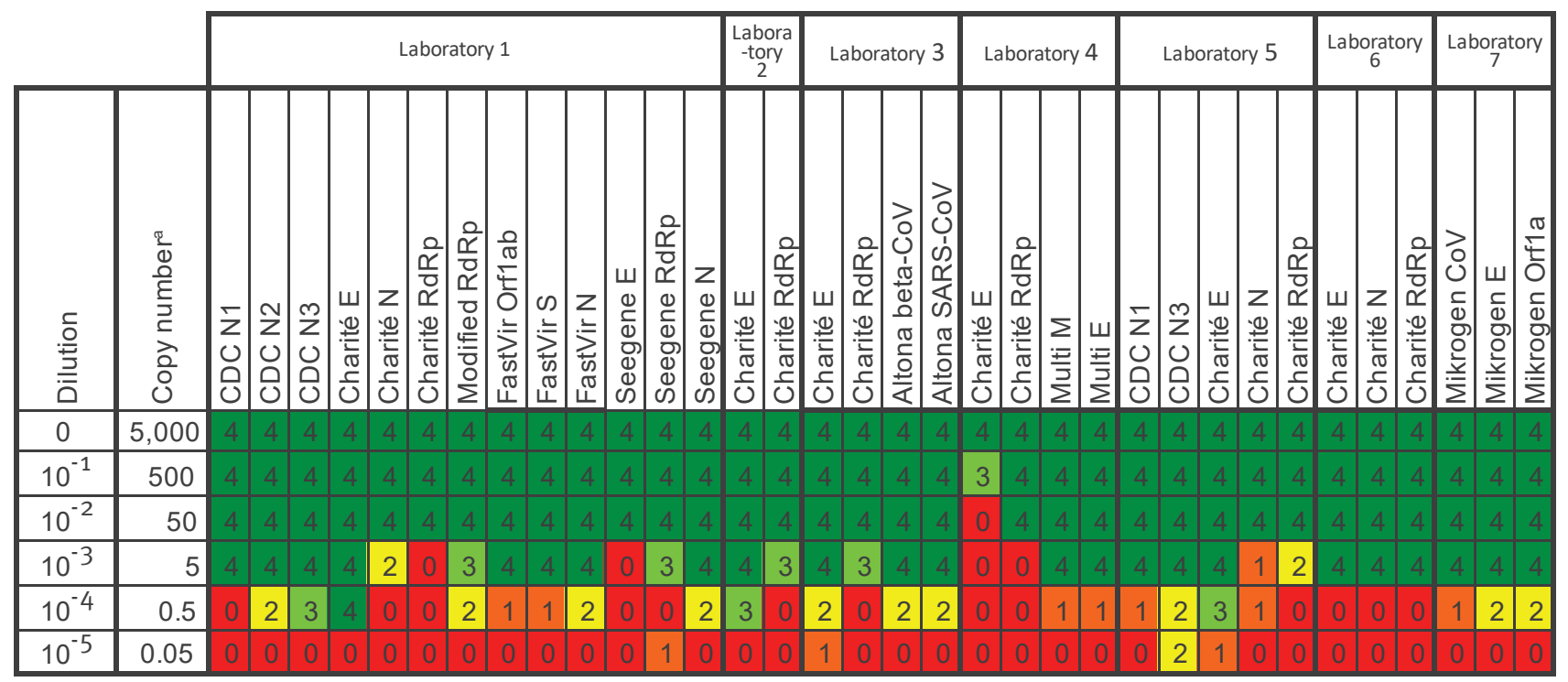

Beta-CoV: Betacoronavirus; CDC: Centers for Disease Control and Prevention; E: envelope gene; N: nucleocapsid gene; ORF: open reading frame; RdRp: RNA-dependent RNA-polymerase gene; S: spike gene; SARS-CoV-2: severe acute respiratory syndrome coronavirus 2.

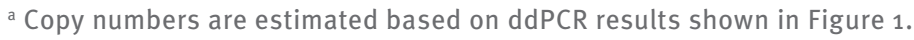

A 1:10 dilution series was prepared from pooled eluates of one SARS-CoV-2 stool sample and sent to participating laboratories to be tested in quadruplicate. The number of positive replicates is indicated and colour-coded as a heatmap with four positive results shown in green and four negative results shown in red. 
FIGURE 3

RT-PCR results of respiratory samples with low positivity, SARS-CoV-2 detection, Germany, March 2020 ( $\mathrm{n}=28$ samples)

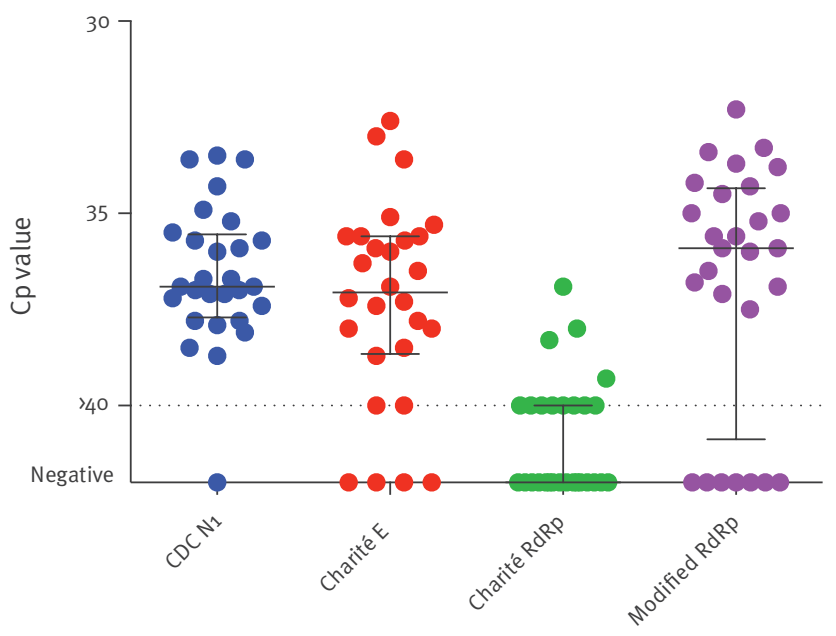

Primer/probe combination

CDC: Centers for Disease Control and Prevention; $\mathrm{Cp}$ : crossing point; E: envelope gene; N: nucleocapsid gene; RdRp: RNAdependent RNA-polymerase gene; SARS-CoV-2: severe acute respiratory syndrome coronavirus 2 .

Nucleic acid eluates of clinical respiratory specimens that initially showed low positive results in the CDC N1 reaction (Cp value > 35) were retested side by side in the CDC N1, the Charité $E$ and original $R d R p$ reaction and using the modified $R d R p$ primers on the Roche LightCycler 480 using the QuantiNova Multiplex RT-PCR kit. Cp values are shown with positive amplifications beyond cycle 40 shown as $>40$ (dotted line).

The undiluted sample showed between 4,325 and 5,015 SARS-CoV-2 RNA copies per reaction using $5 \mu \mathrm{L}$ of eluate for the CDC $N_{1}, N_{2}, N_{3}$ and Charité $E$ protocols, but only 850 and 1,951 RNA copies for the Charité $N$ and $P$ primer/probe combinations (Figure $1 \mathrm{~A})$, respectively, indicating a lower sensitivity of the latter. The 1:10 dilution series displayed good linearity down to a calculated concentration of 0.4 RNA copies per reaction at the $10^{-4}$ dilution for both the CDC $N_{1}$ and $N_{2}$ primer/probe combinations (Figure 1B).

\section{Multicentre and multi-assay comparison}

Result interpretations from the seven participating laboratories are summarised in Figure 2 displaying the number of replicates scored positive by the respective laboratory for each method and dilution. Most methods reliably detected the sample at the $10^{-3}$ dilution, which is equivalent to ca 5 RNA copies for the CDC N1, N2, $N_{3}$ and Charité $E$ reactions based on the absolute quantification by ddPCR. Of note, the Seegene Allplex 2019-nCoV Assay gave negative results for all four replicates in the $E$ gene at the $10^{-3}$ dilution, while reporting positive results for $N$ and $R d R p$ (Laboratory 1). According to the manufacturer's instructions at the time of analysis, this would have been interpreted as an inconclusive result. Of note, the $R d R p$ primer/probe did not show any positive result at the $10^{-4}$ dilution.

\section{Sequence analysis of primer pairs}

Driven by false-negative results for samples with low PCR-positivity using the original Charite $R d R p$ reaction (see below and others $[8,9]$ ), we compared the primer/ probe sequences with currently available SARS-CoV-2 genomes. When compared with all genomes available on GISAID (9,184 SARS-CoV-2 genomes on 15 April 2020, Supplement), the regions used for amplification in the CDC and Charite protocol are highly conserved: Only $1.55 \%, 0.45 \%$ and $2.4 \%$ of genome sequences contain any kind of mismatch within the primer/ probe regions of the CDC $N_{1}, N_{2}$ and $N_{3}$ protocols, respectively, and $0.25 \%, 0.29 \%$ and $0.67 \%$ in the primer/probe regions of the Charite $E, R d R p$ and $N$ pro tocols, respectively.

The Charité $R d R p$ reverse primer contains an ambiguity base at position 15,519 that does not match the reference sequence (Wuhan-Hu-1/2019), with an S (i.e. $\mathrm{G}$ or $\mathrm{C}$ ) instead of $\mathrm{T}$ for the reverse complement (Supplementary Figure S1). The other ambiguity base at 15,528 showing $Y$ (i.e. $C$ or $T$ ) should be changed to $T$ because the currently circulating viruses have a $T$ at this position and no polymorphisms were detected in any of the 9,184 sequences submitted to date (accession date: 15 April 2020). Based on computation using Primer Express v3.0 (Applied Biosystems, Dreieich, Germany) annealing temperatures were predicted to be $64^{\circ} \mathrm{C}$ for the $R d R p$ forward and $51^{\circ} \mathrm{C}$ for the $R d R p$ reverse primer of the Charité protocol. This temperature difference may result in reduced PCR efficiency. To address this issue, modified $R d R p$ primers were synthesised as shown in Supplementary Figure $\mathrm{S} 1$ and tested in comparison with the original primers.

\section{Differential detection of respiratory samples with low $P C R$ positivity}

Testing the dilution series with these modified $R d R p$ primers (see above and Supplementary Figure $\mathrm{S}_{1}$ ) yielded positive results for two additional dilution steps $\left(10^{-3}\right.$ and $\left.10^{-4}\right)$ compared with the original Charité $R d R p$ primers (Figure 1). To further compare the sensitivity of these modified $R d R p$ primers with the original version of the Charite $R d R p$ primers and the Charité $E$ and the $\operatorname{CDC} N 1$ reaction, we retested 28 eluates of clinical respiratory specimens from the diagnostic unit at Laboratory 1 that had shown crossing point (Cp) values>35 in the initial CDC $N_{1}$ reaction. Using the original version of the confirmatory Charité $R d R p$ primers, 16 of 28 samples tested negative, but 11 of these showed positive results using the modified primers (Figure 3). Overall, the detection by the Charite $E$, modified Charite $R d R p$, and CDC N1 reactions were robust. Notably, six and seven of these 28 respiratory samples scored negative or at the limit of detection $(\mathrm{Cp}=40)$ in the Charite $E$ and modified Charité $R d R p$ reactions, while only one sample came up negative in retesting in the CDC N1 reaction $(p=0.04$ and $\mathrm{p}=0.02$, chi-squared-test comparing Charité $E$ and modified $R d R p$ to CDC $N 1$, respectively). Of note, in a routine clinical setting, the $C D C N_{1}$ reaction also 
detected SARS-CoV-2 RNA in nucleic acid extracts from 37 of 83 sera ( $45 \%$ ) from COVID-19 patients in intensive care units, with a positive correlation of their $C p$ values with those of the corresponding respiratory material (Spearman Rank correlation co-efficient $r=0.4285, p$ (two-tailed) $<0.0001$ (data not shown)).

\section{Conclusion}

The majority of RT-PCR assays for SARS-CoV-2 examined in this study detected ca 5 RNA copies per reaction, reflecting a high sensitivity and their suitability for screening purposes world-wide. A reduced sensitivity was noted for the original Charité $R d R p$ gene confirmatory protocol, which may have impacted the confirmation of some COVID-19 cases in the early weeks of the pandemic. The protocol needs to be amended to improve the sensitivity of the $R d R p$ reaction. The CDC $N_{1}$ primer/probe set was sensitive and robust for detection of SARS-CoV-2 in nucleic acid extracts from respiratory material, stool and serum from COVID-19 patients.

\section{Acknowledgements}

We acknowledge the authors, originating and submitting laboratories of the sequences from GISAID's EpiCov database on which this research is based. All submitters of data may be contacted directly via the GISAID website (www.gisaid.org). This work was supported by LMUexcellent funding of LMU München. We thank Mikrogen for participating in this investigator-initiated study.

\section{Conflict of interest}

None declared.

\section{Authors' contributions}

MM, HM, HN, NG-K, SB and OTK designed the study. MM, $H M, D H, N A, R K, M W, S Z$ performed experiments. RW, DH, $A B, H R, A S, B L, S C, C D$, UP analysed data. HB, AG and SK assembled SARS-CoV-2 genome sequences and extracted variants for the analysis of the primer binding regions. MM and OTK wrote the manuscript.

All authors discussed the results and commented on the final manuscript.

\section{References}

1. Wu F, Zhao S, Yu B, Chen YM, Wang W, Song ZG, et al. A new coronavirus associated with human respiratory disease in China. Nature. 2020;579(7798):265-9. https://doi.org/10.1038/ S41586-020-2008-3 PMID: 32015508

2. Centers for Disease Control and Prevention (CDC). Information for laboratories about coronavirus (COVID-19). Atlanta: CDC. [Accessed: 16 April 2020]. Available from: https://www.cdc. gov/coronavirus/2019-ncov/lab/index.html

3. Corman VM, Landt O, Kaiser M, Molenkamp R, Meijer A, Chu DK, et al. Detection of 2019 novel coronavirus (2019-nCoV) by real-time RT-PCR. Euro Surveill. 2020;25(3):2000045. https:// doi.org/10.2807/1560-7917.ES.2020.25.3.2000045 PMID: 31992387

4. World Health Organization (WHO). Coronavirus disease (COVID-19) technical guidance: Laboratory testing for 2019-nCoV in humans. Geneva: WHO. [Accessed: 16 April 2020]. Available from: https://www.who.int/emergencies/ diseases/novel-coronavirus-2019/technical-guidance/ laboratory-guidance

5. van Kasteren PB, van der Veer B, van den Brink S, Wijsman $L$, de Jonge J, van den Brandt $A$, et al. Comparison of seven commercial RT-PCR diagnostic kits for COVID-19. I Clin Virol. 2020;128:104412. https://doi.org/10.1016/j.jcv.2020.104412 PMID: 32416600

6. Konrad R, Eberle U, Dangel A, Treis B, Berger A, Bengs K, et al. Rapid establishment of laboratory diagnostics for the novel coronavirus SARS-CoV-2 in Bavaria, Germany, February 2020. Euro Surveill. 2020;25(9):2000173. https://doi. org/10.2807/1560-7917.ES.2020.25.9.2000173 PMID: 32156330

7. Wolf GK, Glueck T, Huebner J, Muenchhoff M, Hoffmann D, French LE, et al. Clinical and epidemiological features of a family cluster of symptomatic and asymptomatic SARS-CoV-2 infection. J Pediatric Infect Dis Soc. 2020; piaao6o. https://doi. org/10.1093/jpids/piaao6o PMID: 32441753

8. Vogels CBF, Brito AF, Wyllie AL, Fauver JR, Ott IM, Kalinich $C C$, et al. Analytical sensitivity and efficiency comparisons of SARS-COV-2 qRT-PCR primer-probe sets. medRxiv. 2020:2020.03.30.20048108.

9. Nalla AK, Casto AM, Huang MW, Perchetti GA, Sampoleo R, Shrestha L, et al. Comparative performance of SARS-CoV-2 detection assays using seven different primer-probe sets and one assay kit. J Clin Microbiol. 2020;58(6):e00557-20. https:// doi.org/10.1128/JCM.00557-20 PMID: 32269100

License, supplementary material and copyright

This is an open-access article distributed under the terms of the Creative Commons Attribution (CC BY 4.0) Licence. You may share and adapt the material, but must give appropriate credit to the source, provide a link to the licence and indicate if changes were made.

Any supplementary material referenced in the article can be found in the online version.

This article is copyright of the authors or their affiliated institutions, 2020. 